\title{
aBoard: Uma Plataforma para Educação Inclusiva a partir de Comunicação Aumentativa e/ou Alternativa
}

\author{
Natália M. Franco ${ }^{1}$, Thiago P. Lima ${ }^{1}$, Augusto L. Lima ${ }^{1}$, Edson A. Silva ${ }^{1}$, \\ Rinaldo J. Lima ${ }^{1}$, Ticia F. Cavalcante ${ }^{2}$, Robson N. Fidalgo ${ }^{1}$ \\ ${ }^{1}$ Centro de Informática - Universidade Federal de Pernambuco (UFPE) \\ ${ }^{2}$ Centro de Educação - Universidade Federal de Pernambuco (UFPE) \\ Recife - PE - Brasil \\ \{nmf,tpl,all3, eas4,rjl4,rdnf\}@cin.ufpe.br, ticiaferro@gmail.com
}

\begin{abstract}
Speech impaired people needs Inclusive Education (IE) and specific resources for developing their potencial and learning. In this context, this article presents the aBoard Plataform, a free and cloud-based set of softwares for Augmentative and Alternative Communication (AAC) that can be adapted for IE pedagogical activities (e.g., teaching letters, numbers and routines). This plataform is evaluated in comparison to other solutions according to specific functionalities for AAC aplications as well as their adherence to the Nielsen's Heuristics. aBoard stands out from other solutions and is configured as a suitable tool for public-inclusive education context.
\end{abstract}

Resumo. Pessoas com deficiência na comunicação precisam de uma Educação Inclusiva (EI) e de recursos específicos para desenvolver o seu potencial e melhorar a sua aprendizagem. Neste contexto, este artigo apresenta a Plataforma aBoard, um conjunto de softwares gratuitos e em nuvem para Comunicação Aumentativa elou Alternativa (CAA) que pode ser adaptada para atividades pedagógicas da EI (e.g., ensino de letras, números e rotinas). Esta plataforma é avaliada em comparação com soluções similares no que tange a funcionalidades específicas para aplicações de CAA e à aderência às heurísticas de Nielsen. $O$ aBoard se destaca das demais soluções configurando-se como uma ferramenta adequada para o contexto do ensino público inclusivo.

\section{Introdução}

No Brasil, segundo o Censo 2010 do IBGE [IBGE 2010], existem 2,6 milhões de pessoas com alguma deficiência intelectual (e.g., Transtorno do Espectro Autista e Síndrome de Down) ou desordens neurológicas (e.g., Paralisia Cerebral, Lesão Crânio Encefálica e Microcefalia) que podem ter sua capacidade de comunicação limitada de forma temporária ou permanente. De maneira geral, a privação na comunicação restringe as possibilidades de acesso igualitário à educação e às oportunidades de crescimento intelectual. Quando esta privação ocorre durante os primeiros anos de vida, esta pode provocar déficit no desenvolvimento cognitivo e atraso na aquisição da língua escrita [Mousinho et al. 2008]. Para estes casos, faz-se necessária a inserção de Tecnologias Assistivas (TA) que visam complementar a capacidade de comunicação destes indivíduos. Neste contexto, estas TA são denominadas recursos de Comunicação Aumentativa e/ou Alternativa (CAA). 
A CAA é destinada a atender pessoas sem fala ou escrita funcional ou que estão em defasagem entre sua necessidade comunicativa e sua habilidade em falar e/ou escrever [ASHA 2017]. Os recursos de CAA visam reconhecer e valorizar todas as tentativas de comunicação de um indivíduo - sejam elas por meio de gestos, expressões faciais, olhares, escrita ou desenhos -, e tentam complementá-las. Neste cenário, os recursos de CAA são adequados para atender às necessidades básicas de expressão destes indivíduos, uma vez que, por meio da seleção de um conjunto de pictogramas (i.e., desenhos figurativos que representam objetos, ações ou conceitos), estes indivíduos podem estabelecer uma comunicação básica. Na Figura 1, apresenta-se um exemplo de frase construída com pictogramas.

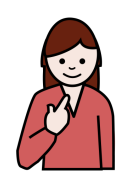

EU
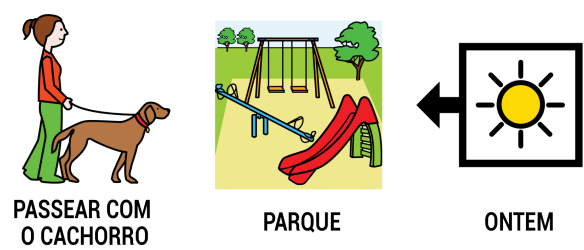

ONTEM

Figura 1. Exemplo de frase com pictogramas

No contexto da educação, as classes comuns do ensino regular não são suficientes para prover assistência compatível com as necessidades destes indivíduos, de tal forma que estas pessoas precisam de uma Educação Inclusiva (EI). Assim, o Ministério da Educação [BRASIL 2009] instituiu que os alunos com deficiência devem ser matriculados nas classes comuns do ensino regular, bem como no Atendimento Educacional Especializado (AEE). O AEE tem como função, complementar ou suplementar a formação do aluno por meio da disponibilização de recursos que eliminem as barreiras para sua plena participação na sociedade e que ajudem no desenvolvimento de sua aprendizagem. Neste contexto, o professor precisa realizar adequações na prática pedagógica para que os alunos consigam participar das situações cotidianas da sala de aula a partir de um canal alternativo àquele que está impedido. Estas adequações podem ser feitas utilizando uma abordagem gameficada [Farias et al. 2015, Moura et al. 2016] ou, mais focada na comunicação (i.e., com recursos de CAA).

Os recursos de CAA são importantes para as pessoas com deficiência na fala. Porém, apesar de existirem várias soluções para CAA, estas não se adequam bem ao contexto nacional do ensino público inclusivo, visto que, em sua maioria, se apresentam na língua inglesa e/ou não são gratuitas. Estas características impõem barreiras na adoção das soluções, pois, professores e alunos não dominam o inglês, tampouco as escolas dispõem de recursos para financiar a compra de licenças de software. Neste contexto, este trabalho apresenta uma plataforma computacional gratuita e em nuvem para CAA adequada ao contexto nacional do ensino público inclusivo, a qual é denominada aBoard.

A Plataforma aBoard tem como diferencial o fato de ser em nuvem e por ter as funcionalidades de sugerir símbolos e oferecer respostas rápidas. $\mathrm{O}$ fato de ser em nuvem permite que o usuário tenha acesso aos seus dados a partir de qualquer dispositivo. Com relação as funcionalidades, estas se destacam, respectivamente, pela capacidade de sugerir pictogramas de acordo com o elemento previamente selecionado e pela facilidade de usar expressões básicas (e.g., sim, não, por favor, obrigado) sem interferir na construção de uma expressão. Além disso, o aBoard provê uma plataforma que: 1) É disponível 
offline, não necessitando de conexão com a Internet para utilizar o dispositivo móvel; 2) Tem uma interface minimalista, o que mantém o foco do usuário no que é importante - os pictogramas; 3) Organiza o conteúdo em categorias, o que agiliza a seleção dos pictogramas, pois reduz o espaço de busca; 4) É em nuvem, permitindo o acesso aos seus dados a partir de qualquer dispositivo; 5) Provê feedback visual e sonoro ao selecionar qualquer pictograma ou botão de ação, funcionando como um reforço para o usuário; 6) Permite a criação explícita de expressões (e.g., frases e operações matemáticas); e 7) Permite a personalização do conteúdo, o que aumenta a identificação entre usuário e dispositivo, encorajando a sua adoção. De modo a mostrar resultados práticos sobre o uso da plataforma no contexto da EI, apresenta-se como esta pode ser usada para adaptar diferentes atividades pedagógicas inclusivas. Além disso, a fim de obter evidências de que o trabalho proposto avança o estado da arte, a Plataforma aBoard é comparada com outras soluções gratuitas e com versão em português, no que se refere às Heurísticas de Nielsen [Nielsen 1995].

O restante deste artigo está organizado da seguinte forma: na Seção 2 apresentase a visão geral e as tecnologias da Plataforma aBoard com o seu potencial de adaptação de conteúdos; na Seção 3 apresenta-se a avaliação comparativa da Plataforma aBoard com as demais soluções computacionais, desde a seleção dos critérios de avaliação até a compilação dos resultados; e, finalmente, na Seção 4 apresentam-se as considerações finais deste artigo, bem como os trabalhos futuros.

\section{Plataforma aBoard}

A Plataforma aBoard é formada por três componentes de software: 1) aplicativo aBoard; 2) aBoard Editor; e 3) aBoard Server. O aplicativo visa permitir que pessoas com deficiência na fala possam, a partir da seleção de pictogramas em um dispositivo móvel, produzir frases que expressem seus desejos, opiniões e sentimentos, aumentando a interação social e reduzindo a frustração desses indivíduos e realizar atividades pedagógicas adaptadas (e.g., contação de histórias e construção de expressões matemáticas). Com o propósito de agilizar e facilitar estas construções/produções, o aplicativo aBoard oferece os recursos de sugestão de pictogramas e respostas rápidas. A partir do primeiro recurso, o usuário recebe como sugestão uma lista de pictogramas que podem completar o sentido do último pictograma selecionado. Por exemplo, após a seleção do pictograma "Comer", são sugeridos pictogramas referentes a coisas "comestíveis", de tal forma que o usuário não perde tempo com pictogramas de coisas "bebíveis". Por sua vez, o recurso de respostas rápidas permite, a partir de expressões básicas e frequentes (i.e., sim, não, por favor, obrigado, desculpa, ajuda, olá, até logo, não sei e tanto faz), estabelecer um diálogo paralelo sem interferir na construção/produção de uma expressão. Por exemplo, durante a construção de uma expressão, o usuário pode usar o recurso de resposta rápida para solicitar ajuda ou confirmar/negar uma pergunta feita pelo mediador (i.e., familiares e profissionais de educação/saúde). O aplicativo aBoard encontra-se na loja da Google a partir do link https://play.google.com/store/apps/details?id=assistive. aboard e o seu tutorial de uso pode ser encontrado em: http: / / assistive.cin. ufpe.br/pt/aboard-app/_intro/Tutorial_aBoard.pdf. Por sua vez, o aBoard Editor é um sistema Web que provê aos mediadores das pessoas com deficiência na fala um conjunto de funcionalidades que permitem organizar, criar, editar e excluir os pictogramas que formam o conteúdo (i.e, vocabulário e atividades pedagógicas) utilizado 
VI Congresso Brasileiro de Informática na Educação (CBIE 2017)

Anais do XXVIII Simpósio Brasileiro de Informática na Educação (SBIE 2017)

no aplicativo aBoard. Ou seja, o aBoard Editor personaliza o conteúdo a ser exibido no aplicativo aBoard. Vale destacar que os pictogramas são organizados a partir dos conceitos de categorias e elementos, os quais se baseiam na ideia de pastas e arquivos de um computador, respectivamente. Ou seja, as categorias podem ter subcategorias e/ou elementos. Além disso, vale ressaltar que o aBoard Editor disponibiliza aproximadamente 15 mil pictogramas do ARASAAC [Palao 2017] para a personalização do conteúdo. O aBoard Editor está disponível a partir do site http: / / assistive.cin.ufpe.br / aboard/ e o seu tutorial de uso pode ser encontrado em: http://assistive.cin. ufpe.br/pt/editor/_intro/Tutorial_aBoard_Editor.pdf. Na Figura 2 mostram-se as interfaces gráficas do sistema Web aBoard Editor e do aplicativo aBoard. Por fim, o aBoard Server é um serviço em nuvem responsável pelo armazenamento dos dados, disponibilização do conteúdo e controle de acesso dos usuários.
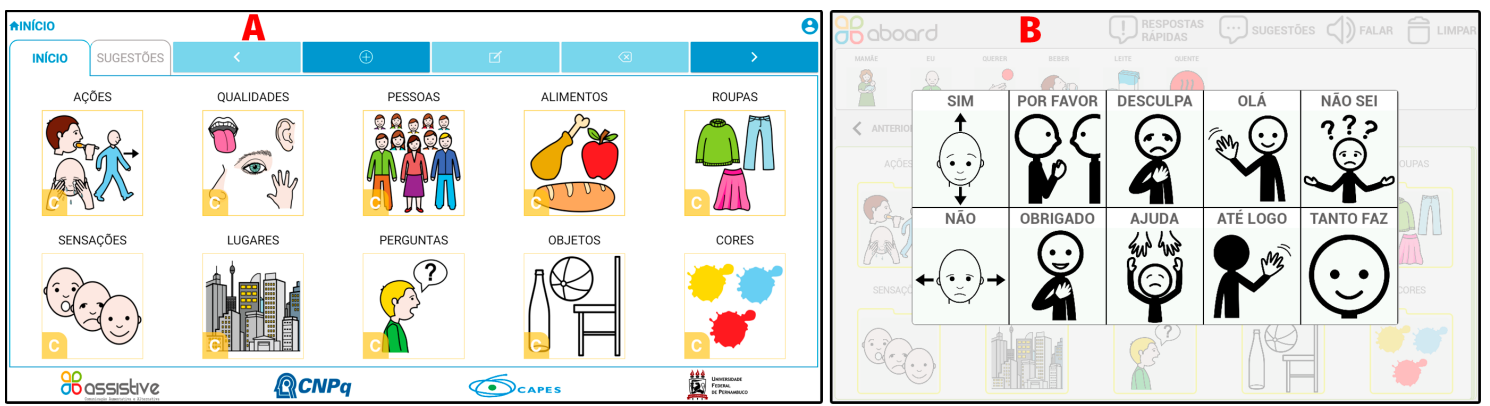

Figura 2. Interfaces (A) do sistema Web aBoard Editor e (B) do aplicativo aBoard

Na Figura 3 apresentam-se os componentes, tecnologias e tipos de usuários da Plataforma aBoard. O aplicativo aBoard é desenvolvido utilizando a linguagem JavaScript em conjunto com o framework Titanium, pois este tem facilidades para gerar aplicativos Android e iOS. O aBoard Editor é desenvolvido com o framework Angular 2, seguindo as especificações HTML5 e CSS3. Por fim, o serviço aBoard Server é desenvolvido utilizando a linguagem Java, com o framework SpringBoot para controle do fluxo de serviço, o padrão JSON Web Token para controle de acesso e o PostgreSQL para gerenciar os dados armazenados.

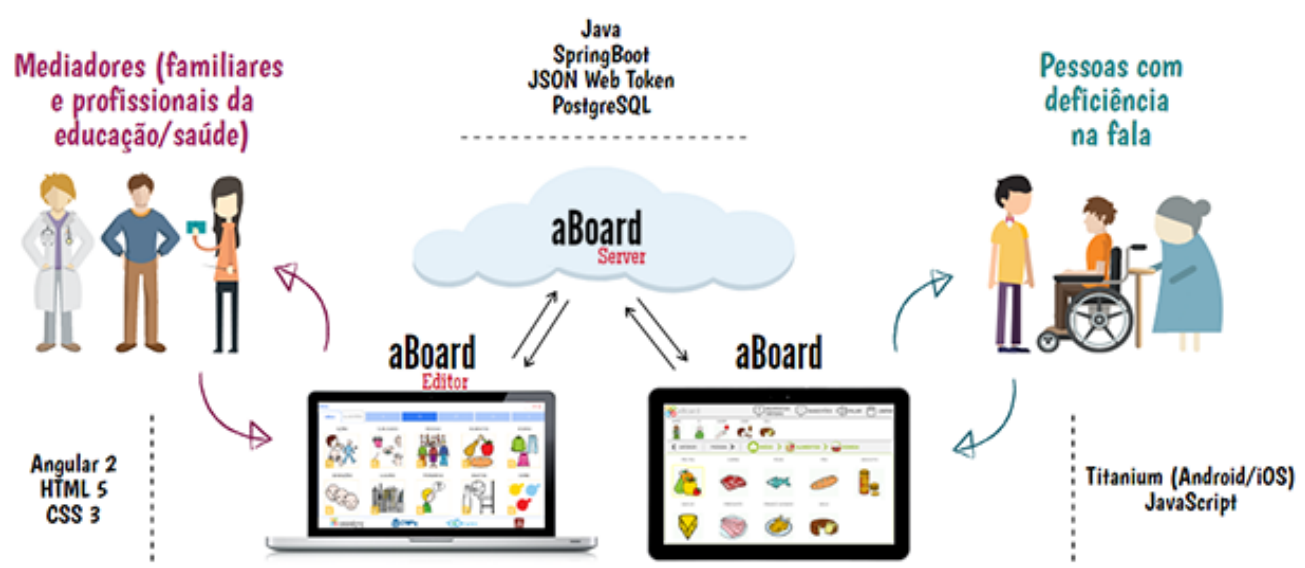

Figura 3. Componentes, tecnologias e tipos de usuários da Plataforma aBoard 
VI Congresso Brasileiro de Informática na Educação (CBIE 2017)

Anais do XXVIII Simpósio Brasileiro de Informática na Educação (SBIE 2017)

\subsection{Uso da Plataforma aBoard para educação inclusiva}

Dada a capacidade de personalização de conteúdo, a Plataforma aBoard pode ser usada por professores da educação inclusiva para diversos fins, tais como: 1) Adaptação de conteúdos pedagógicos - e.g., ensino de letras, sílabas, números e conceitos como animais, cores e lugares; 2) Instrução de rotinas - e.g., a sequência de ações para lavar as mãos; e 3) Atividades lúdicas - e.g., contação de histórias e reprodução de músicas. Na Figura 4 apresentam-se quatro adaptações feitas com a Plataforma $a$ Board que podem ser utilizadas em atividades pedagógicas com crianças:

1. Na adaptação apresentada na Figura 4-A tem-se um exemplo de como trabalhar as letras do alfabeto de maneira visual e contextualizada;

2. Na adaptação apresentada na Figura 4-B tem-se um exemplo de como trabalhar as operações básicas de matemática;

3. Na adaptação apresentada na Figura 4-C tem-se um exemplo de como trabalhar conceitos usando músicas infantis. No caso do exemplo, apresenta-se a adaptação da música "Sopa", de autoria do Palavra Cantada;

4. Na adaptação apresentada na Figura 4-D tem-se um exemplo de como trabalhar rotinas, ensinando os passos necessários para a realização de tarefas. No caso do exemplo, aborda-se a sequência de passos envolvidos na atividade de "Lavar as Mãos".
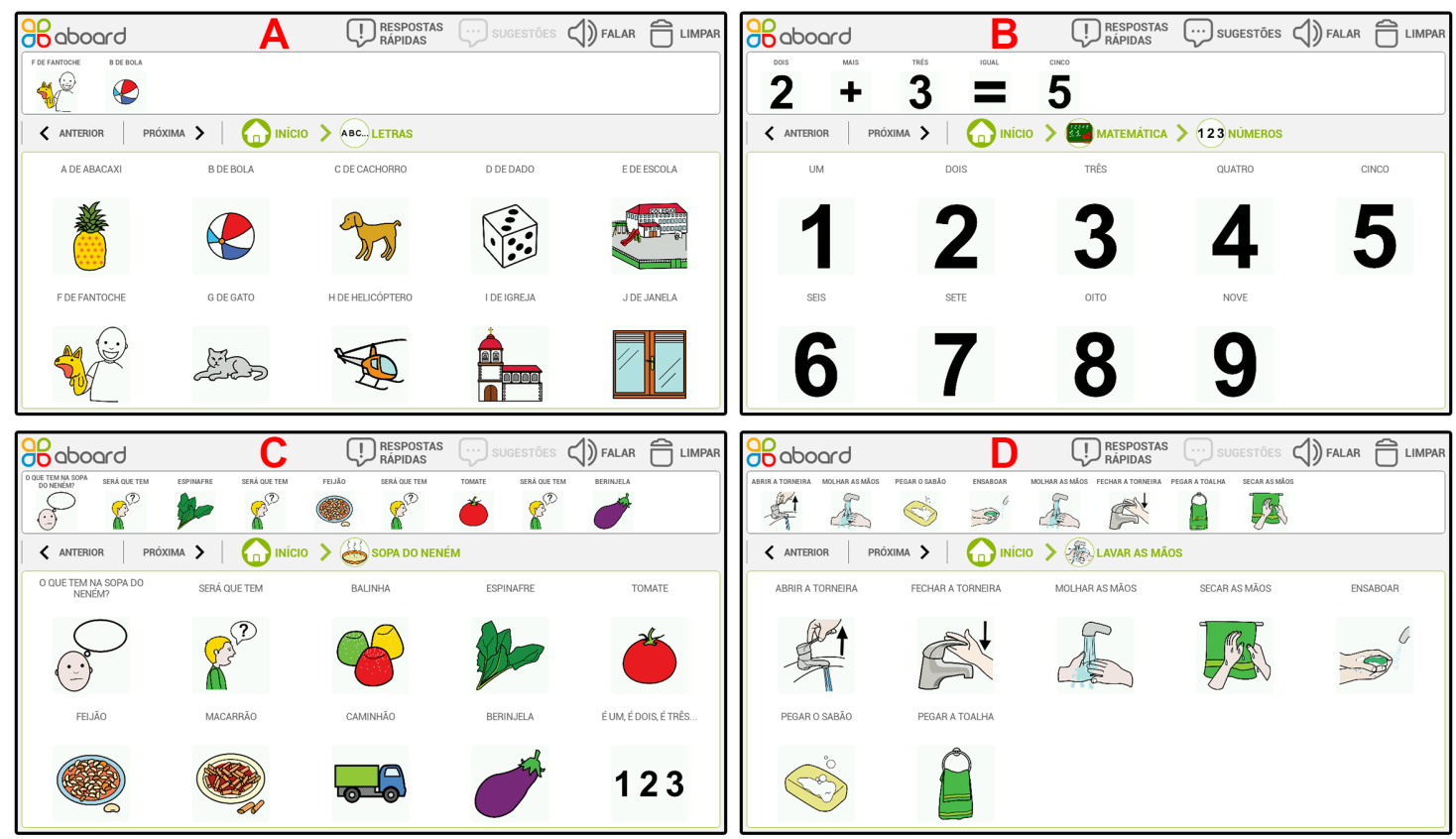

Figura 4. Adaptação de atividades pedagógicas com a Plataforma aBoard

A ideia deste tipo de adaptação é explorar novos conceitos a partir de um recurso lúdico. Ressalta-se que o limite desta tecnologia é a criatividade e o empenho dos professores em adaptar conteúdos e explorar o potencial de cada aluno. Além disso, destacase que, pelo fato da Plataforma aBoard ser na nuvem, o conteúdo pode ser criado em qualquer computador com acesso à Internet (não é necessário instalar nenhum software para isto) e usado em um dispositivo móvel com o aplicativo aBoard instalado. Note 
que a personalização do conteúdo requer acesso constante à Internet. Contudo, o uso do conteúdo no dispositivo móvel pode ser offline, pois a Internet só é necessária quando se deseja sincronizar o conteúdo produzido no computador com o conteúdo a ser exibido no dispositivo móvel. Assim, mesmo que o computador ou o dispositivo móvel sejam danificados, o conteúdo guardado na nuvem sempre poderá ser acessado em qualquer outro computador ou dispositivo móvel.

\section{Avaliação comparativa}

\subsection{Trabalhos Relacionados}

Considerando o contexto nacional do ensino público inclusivo, além de ser um aplicativo gratuito e com versão em português, foram considerados os seguintes critérios para selecionar os trabalhos relacionados: 1) Estar disponível na loja da Google - este critério visa garantir que o aplicativo pode ser facilmente encontrado e executado em dispositivos móveis de baixo custo; 2) Ter mais de 1.000 downloads e nota maior ou igual a quatro estrelas - este critério visa garantir que o aplicativo tem uma boa qualidade; 3) Permitir personalização de conteúdo e criação de expressões - este critério visa garantir a edição do conteúdo e a construção explícita de expressões e diálogos. Apenas dois aplicativos atenderam a estes critérios: Adapt ${ }^{1}$ e LetMeTalk ${ }^{2}$, os quais não têm artigos científicos.

O aplicativo Adapt tem de 10.000 à 50.000 instalações e encontra-se em sua versão beta 0.7. Este aplicativo não requer uma conta de usuário para ser usado e, ao iniciá-lo, este já vem com um conjunto de pictogramas para uso. As principais funcionalidades disponíveis são: rolagem do conteúdo por paginação, sugestão ou predição de pictogramas e rolagem da tela por botão. Já o aplicativo LetMeTalk tem de 100.000 à 500.000 instalações e atualmente está na versão 1.4.29. Este aplicativo também não requer a criação de uma conta de usuário para ser usado e, ao iniciá-lo, já é mostrado um conjunto de pictogramas organizados em categorias. As principais funcionalidades disponíveis são: disponibilidade de cópia de conteúdo, alteração da posição de qualquer pictograma na área de conteúdo, imagem customizada, expressão com pictogramas e suas legendas, reprodução da expressão toda ou de um pictograma desta, compartilhamento das expressões construídas, navegação visível e livre. Uma visão mais detalhada dessas funcionalidades é apresentada na próxima seção.

\subsection{Materiais e métodos}

Para esta avaliação foi realizado um focus group [Shull et al. 2008] com 4 profissionais que trabalham há pelo menos 1 ano com CAA e 1 pesquisador mediador. Este método foi escolhido pois permite obter, em pouco tempo, múltiplos pontos de vista de um grupo de especialistas em uma área específica. Outra característica que contribuiu para a escolha deste método foi a possibilidade de intervenção do pesquisador mediador durante a sessão, permitindo assim o direcionamento das discussões e a elucidação de dúvidas.

O primeiro objetivo do focus group foi definir os critérios de avaliação. Assim, com a ajuda dos profissionais e da mediação do pesquisador, definiu-se um conjunto de 3 características básicas para aplicações de CAA, a saber: 1) Organização do Conteúdo -

\footnotetext{
${ }^{1}$ https://play.google.com/store/apps/details?id=com.argulu.adapt

${ }^{2}$ https://play.google.com/store/apps/details?id=de.appnotize.letmetalk
} 
VI Congresso Brasileiro de Informática na Educação (CBIE 2017)

Anais do XXVIII Simpósio Brasileiro de Informática na Educação (SBIE 2017)

maneira de estruturar os pictogramas; 2) Construção de Expressões - maneira de estabelecer a comunicação; e 3) Navegação pelo Conteúdo - maneira de percorrer pelo conteúdo. Em seguida, para cada característica, foram elencadas funcionalidades importantes para aplicações de CAA, as quais podem ser vistas na Tabela 1 e são explicadas a seguir: (1) Legenda na parte superior do pictograma - permite que a mão do utilizador não encubra a legenda quando o pictograma é selecionado; (2) Disponibilidade de cópia de conteúdo - permite acessar uma cópia do conteúdo a partir de diferentes dispositivos; (3) Alteração da posição de qualquer pictograma na área de conteúdo - permite organizar a área de conteúdo da forma que for mais conveniente/confortável para o usuário; (4) Imagem customizada - permite a inclusão de imagens (i.e., fotos tiradas pelos dispositivos móveis, imagens obtidas na Internet, etc.) que sejam familiares ao usuário; (5) Áudio customizado - permite a inclusão de áudios (e.g., gravações de falas e músicas) familiares ao usuário; (6) Rolagem de conteúdo por paginação - permite que os pictogramas estejam sempre visíveis, pois, diferente da rolagem do tipo infinita, a rolagem por paginação garante que os pictogramas nunca serão cortados; (7) Comunicação paralela à construção da expressão - permite que o usuário consiga dialogar com o seu interlocutor sem que a estrutura da expressão em construção seja modificada e/ou perdida; (8) Sugestão ou predição de pictogramas - possui algum recurso para sugerir/predizer pictogramas; (9) Interface simples - possui interface compreensível ao usuário; (10) Tutorial e ajuda disponíveis - disponibiliza material de apoio ao usuário; (11) Expressão com pictogramas e suas legendas - indica que os pictogramas da expressão são exibidos junto com as suas legendas; (12) Reprodução da expressão toda - permite vocalizar as expressões construídas; (13) Reprodução isolada de qualquer pictograma da expressão - permite reforçar um pictograma específico da expressão; (14) Compartilhamento da expressão construída - permite postar as expressões em mídias sociais e em aplicativos de mensagens instantâneas; (15) Exclusão da expressão toda - permite limpar por completo uma expressão construída com apenas uma ação; (16) Exclusão de um pictograma qualquer da expressão - permite excluir qualquer pictograma e não apenas o último; (17) Alteração da posição de um pictograma da expressão - permite reordenar os pictogramas da expressão em construção; (18) Navegação sempre visível - tem recurso de navegação semelhante ao breadcrumb; (19) Distinção entre categoria e elemento - permite distinguir facilmente entre um pictograma que representa um conjunto de elementos e um elemento, respectivamente; (20) Rolagem da tela por botão - permite a navegação a partir de botões o que facilita o uso do aplicativo por indivíduos sem o movimento de deslizar; (21) Rolagem da tela por swipe - permite a navegação por gestos de deslizar o dedo na tela; (22) Navegação livre - permite construir uma expressão sem seguir uma ordem pré-definida; e (23) Uso intuitivo permite realizar atividades sem que seja necessário decorar os passos para isto.

Uma vez definidas as funcionalidades, estas foram mapeadas para as 10 heurísticas propostas por Nielsen [Nielsen 1995], as quais correspondem a princípios gerais do design de interfaces do usuário e são exibidas na Tabela 2. Ressalta-se que este mapeamento permite a avaliação das Heurísticas de Nielsen, no contexto das aplicações de CAA, de maneira mais objetiva. As características, funcionalidades e heurísticas podem ser observadas na Tabela 1.

Por fim, a Plataforma aBoard foi comparada com as outras soluções gratuitas e em português no que tange a verificação das características e funcionalidades especificadas, bem como no que tange à aderência às Heurísticas de Nielsen. Para isso, durante a 
VI Congresso Brasileiro de Informática na Educação (CBIE 2017)

Anais do XXVIII Simpósio Brasileiro de Informática na Educação (SBIE 2017)

realização do focus group os profissionais interagiram com os aplicativos e, a partir das funcionalidades elencadas, avaliaram, em comum acordo, quais delas estavam presentes em cada aplicativo.

\subsection{Resultados}

$\mathrm{Na}$ Tabela 1 apresenta-se o resultado da avaliação comparativa. Na característica Conteúdo são agrupadas 10 funcionalidades, das quais o Adapt satisfaz 3 (30\%), o LetMeTalk, 5 (50\%) e o aBoard, 7 (70\%). Na característica Expressão são agrupadas 7 funcionalidades, das quais o Adapt não satisfaz nenhuma (0\%), o LetMeTalk, $5(71,4 \%) \mathrm{e}$ o aBoard, 5 (71,4\%). Na característica Navegação são agrupadas 6 funcionalidades, das quais o Adapt satisfaz 2 (33,3\%), o LetMeTalk, 5 (83,3\%) e o aBoard, 6 (100\%). Por fim, no geral, o Adapt satisfaz 5 (21,7\%) dos critérios, o LetMeTalk, 15 (65,2\%) e o aBoard, $18(78,3 \%)$.

Tabela 1. Avaliação comparativa das soluções

\begin{tabular}{|c|c|c|c|c|c|c|}
\hline \# & Característica & Heurística & Funcionalidade & Adapt & LetMeTalk & aBoard \\
\hline 1 & Conteúdo & 1 & Legendas na parte superior do pictograma & Não & Não & Sim \\
\hline 2 & Conteúdo & 2 & Disponibilidade de cópia de conteúdo & Não & Sim & Sim \\
\hline 3 & Conteúdo & 3 & Alteração da posição de qualquer pictograma na área de conteúdo & Não & Sim & Não \\
\hline 4 & Conteúdo & 3 & Imagem customizada & Não & Sim & Não \\
\hline 5 & Conteúdo & 3 & Áudio customizado & Não & Não & Não \\
\hline 6 & Conteúdo & 4 & Rolagem do conteúdo por paginação & Sim & Não & Sim \\
\hline 7 & Conteúdo & 7 & Comunicação paralela à construção da expressão & Não & Não & Sim \\
\hline 8 & Conteúdo & 7 & Sugestão ou predição de pictogramas & Sim & Não & Sim \\
\hline 9 & Conteúdo & 8 & Interface simples & Sim & Sim & Sim \\
\hline \multirow[t]{3}{*}{10} & Conteúdo & 10 & Tutorial e ajuda disponíveis & Não & Sim & Sim \\
\hline & & & Sim & $3(30 \%)$ & $5(50 \%)$ & $7(70 \%)$ \\
\hline & & & Não & $7(70 \%)$ & $5(50 \%)$ & $3(30 \%)$ \\
\hline 11 & Expressão & 1 & Expressões com pictogramas e suas legendas & Não & Sim & Sim \\
\hline 12 & Expressão & 2 & Reprodução da expressão toda & Não & Sim & Sim \\
\hline 13 & Expressão & 2 & Reprodução isolada de qualquer pictograma da expressão & Não & Sim & Sim \\
\hline 14 & Expressão & 2 & Compartilhamento da expressão construída & Não & Sim & Não \\
\hline 15 & Expressão & 3 & Exclusão da expressão toda & Não & Não & Sim \\
\hline 16 & Expressão & 3 & Exclusão de um pictograma da expressão & Não & Sim & Sim \\
\hline \multirow[t]{3}{*}{17} & Expressão & 3 & Alteração da posição de um pictograma na expressão & Não & Não & Não \\
\hline & & & Sim & $0(0 \%)$ & $5(71,4 \%)$ & $5(71,4 \%)$ \\
\hline & & & Não & $7(100 \%)$ & $2(28,6 \%)$ & $2(28,6 \%)$ \\
\hline 18 & Navegação & 1 & Navegação sempre visível & Não & Sim & Sim \\
\hline 19 & Navegação & 1 & Distinção entre categorias e elementos & Não & Sim & Sim \\
\hline 20 & Navegação & 2 & Rolagem da tela por botão & Sim & Não & Sim \\
\hline 21 & Navegação & 2 & Rolagem da tela por swipe & Não & Sim & Sim \\
\hline 22 & Navegação & 3 & Navegação livre & Não & Sim & Sim \\
\hline \multirow[t]{5}{*}{23} & Navegação & 6 & Uso intuitivo & Sim & Sim & Sim \\
\hline & & & Sim & $2(33,3 \%)$ & $5(83,3 \%)$ & $6(100 \%)$ \\
\hline & & & Não & $4(66,7 \%)$ & $1(16,7 \%)$ & $0(0 \%)$ \\
\hline & & & Total Sim & $5(21,7 \%)$ & $15(65,2 \%)$ & $18(78,3 \%)$ \\
\hline & & & Total Não & $18(78,3 \%)$ & $8(34,8 \%)$ & $5(21,7 \%)$ \\
\hline
\end{tabular}

Com base nos resultados apresentados na Tabela 1, pode-se afirmar que: 1) Apenas as funcionalidades 9 (interface simples) e 23 (uso intuitivo) são contempladas por todas as soluções. Tais funcionalidades indicam que estes aplicativos têm uma interface minimalista e que sua utilização ocorre sem que se precise decorar os passos necessários para cada ação; 2) As funcionalidades 5 (áudio customizado) e 17 (alteração da posição de um pictograma na expressão) não são contempladas por nenhuma das soluções. Possuir essas funcionalidades permitiria um feedback sonoro com áudio familiar e mais agradável ao utilizador, bem como uma melhor organização na disposição dos pictogramas para a 
VI Congresso Brasileiro de Informática na Educação (CBIE 2017)

Anais do XXVIII Simpósio Brasileiro de Informática na Educação (SBIE 2017)

construção e correção de expressões, permitindo menor esforço e maior flexibilidade para reordenar os pictogramas de uma expressão; 3) As funcionalidades 3 (alteração da posição de qualquer pictograma na área de conteúdo), 4 (imagem customizada) e 14 (compartilhamento da expressão construída) são implementadas apenas pelo LetMeTalk, o que caracteriza estas funcionalidades como seu diferencial; e 4) As funcionalidades 1 (legenda na parte superior do pictograma), 7 (comunicação paralela à construção da expressão) e 15 (exclusão da expressão toda) são implementadas apenas pelo aplicativo aBoard, denotando que estas funcionalidades são seu diferencial. Vale ressaltar que o Adapt não se destaca diante das demais aplicações com relação a nenhuma funcionalidade.

Na Tabela 2 apresenta-se a sumarização destes resultados em relação à aderência das soluções a cada uma das Heurísticas de Nielsen. No que tange às funcionalidades mapeadas a cada heurística na Tabela 1, tem-se que as heurísticas 6 e 8 são totalmente aderidas por todas as soluções. O Adapt adere totalmente às heurísticas 4, 6 e 8, o LetMeTalk, às heurísticas 6,8 e 10 e, o aBoard, às heurísticas 1, 4, 6, 7, 8 e 10. Vale ressaltar que não foi possível avaliar as heurísticas 5 e 9 , uma vez que não houve a ocorrência de erros no uso dos aplicativos.

Tabela 2. Aderência das soluções às heurísticas de Nielsen

\begin{tabular}{clccc}
\hline$\#$ & Heurística & Adapt & LetMeTalk & aBoard \\
\hline 1 & Visibilidade do estado do sistema & $0 \%$ & $75 \%$ & $100 \%$ \\
2 & Correspondência entre o sistema e o mundo real & $16,6 \%$ & $83,3 \%$ & $83,3 \%$ \\
3 & Controle do usuário e liberdade & $14,28 \%$ & $57,14 \%$ & $42,86 \%$ \\
4 & Consistência e padrões & $100 \%$ & $0 \%$ & $100 \%$ \\
5 & Prevenção de erros & Não avaliada & Não avaliada & Não avaliada \\
6 & Reconhecimento ao invés de recordação & $100 \%$ & $100 \%$ & $100 \%$ \\
7 & Flexibilidade e eficiência de utilização & $50 \%$ & $0 \%$ & $100 \%$ \\
8 & Estética e design minimalista & $100 \%$ & $100 \%$ & $100 \%$ \\
9 & Reconhecimento, diagnóstico e recuperação de erros & Não avaliada & Não avaliada & Não avaliada \\
10 & Ajuda e documentação & $0 \%$ & $100 \%$ & $100 \%$
\end{tabular}

\section{Considerações finais}

Este artigo apresentou a Plataforma aBoard, um conjunto de softwares (aplicativo aBoard, aBoard Editor e aBoard Server) para CAA e EI que tem como pontos fortes o fato de ser em nuvem e ter as funcionalidades de sugestão de pictogramas e respostas rápidas. Esta plataforma é comparada com outras soluções gratuitas e com versão em português no que se refere à aderência às Heurísticas de Nielsen e a funcionalidades desejáveis para um aplicativo de CAA. Neste contexto, a Plataforma aBoard se destaca dos demais aplicativos por implementar um número maior de funcionalidades, bem como pela sua maior aderência às Heurísticas de Nielsen.

Os benefícios que podem ser obtidos a partir do uso da Plataforma aBoard só dependem da criatividade e empenho dos professores em adaptar conteúdos e explorar o potencial de cada aluno. De forma geral, o uso da Plataforma aBoard favorece: 1) A interação do usuário com outras pessoas; 2) $\mathrm{O}$ desenvolvimento de habilidades cognitivas referentes a percepção, atenção, memória (imediata, recente/mediata, remota, visual, auditiva e viso motora), raciocínio, conceituação, linguagem e alfabetização; 3 ) O ensino de rotinas escolares e familiares; 4) A redução de frustrações; 5) A melhora da autoestima e da qualidade de vida do usuário e de seus familiares; 6) A expressão de necessidades, 
VI Congresso Brasileiro de Informática na Educação (CBIE 2017)

Anais do XXVIII Simpósio Brasileiro de Informática na Educação (SBIE 2017)

sentimentos e opiniões de forma mais clara; e 7) A realização de atividades de forma mais independente.

Como projetos futuros, pretende-se dar suporte a todas as funcionalidades da Tabela 1. Vale destacar que, já estão em desenvolvimento: 1) Um acionador externo - para pessoas que tenham alguma limitação motora que as impeça de utilizar um dispositivo móvel -, uma vez que a navegação do aBoard também é baseada em botões; 2) A versão IOS do aBoard; e 3) Um módulo de predição de pictogramas que considera os aspectos sintáticos e semânticos das expressões produzidas. Ressalta-se que o objetivo deste módulo é servir de apoio ao letramento e à alfabetização, uma vez que ensina aos alunos a maneira correta de estruturar as frases.

\section{Agradecimentos}

Esta pesquisa é apoiada pelo CNPq (458798/2013-4 e 461492/2014-8) e pela Capes (88887.095638/2015-01).

\section{Referências}

ASHA (2017). American Speech-Language-Hearing Association. http://www.asha.org/.

BRASIL (2009). Resolução no 4, de 2 de Outubro de 2009. Institui Diretrizes Operacionais para o Atendimento Educacional Especializado na Educação Básica, modalidade Educação Especial.

Farias, E., Cunha, M., and Souza, J. W. (2015). Abc autismo? uma aplicação mobile para auxiliar no processo alfabetizador de crianças com autismo. In Anais dos Workshops do Congresso Brasileiro de Informática na Educação, volume 4, page 232.

IBGE, C. D. (2010). Características gerais da população, religião e pessoas com deficiência. Rio de Janeiro: Instituto Brasileiro de Geografia e Estatística.

Moura, D., de Oliveira Filho, D. L. S., Laertius, D., Silva, A. J. G., Paiva, P., de Sales, T., Cavalcante, R., and Queiroz, F. (2016). Teo: Uma suíte de jogos interativos para apoio ao tratamento de crianças com autismo. In Simpósio Brasileiro de Informática na Educação-SBIE, volume 27, page 627.

Mousinho, R., Schmid, E., Pereira, J., Lyra, L., Mendes, L., and Nóbrega, V. (2008). Aquisição e desenvolvimento da linguagem : dificuldades que podem surgir neste percurso. Revista de Psicopedagogia, 25(78):297-306.

Nielsen, J. (1995). 10 Usability Heuristics for User Interface Design. http://www.nngroup.com/articles/ten-usability-heuristics/.

Palao, S. (2017). ARASAAC - Portal Aragonês de Comunicação Aumentativa e Alternativa. http://catedu.es/arasaac/.

Shull, F., Singer, J., and Sjoberg, D. I. K. (2008). Guide to Advanced Empirical Software Engineering. 